\title{
Anaplastic Astrocytoma Presenting as Ischemic Stroke: A Diagnostic Pitfall
}

\author{
Krishnaiah B* and Ermak D \\ Department of Neurology, Penn State Milton S. Hershey \\ Medical Center, USA \\ *Corresponding author: Balaji Krishnaiah, \\ Department of Neurology, Penn State Milton S. Hershey \\ Medical Center, 30 Hope Drive, EC 037, Hershey, PA \\ 17033, USA
}

Received: April 05, 2017; Accepted: May 08, 2017; Published: May 18, 2017

\begin{abstract}
Gliomas account for the great majority of primary tumors that arise within the brain parenchyma. The term "glioma" refers to tumors that have histologic features similar to normal glial cells. Anaplastic astrocytomas fall under the category of high grade gliomas, WHO grade III. Most common acute presentation of brain tumor includes intracranial bleed, seizures or focal neurological deficit and rarely as acute ischemic stroke. We are presenting a case of anaplastic astrocytoma who presented with acute onset stroke like symptoms.
\end{abstract}

Keywords: Stroke; Astrocytoma; Diagnostic pitfall

\section{Case Presentation}

This is the case of a 68 year old male with past medical history significant for hypertension and dyslipidemia who presented initially to an outside facility for confusion and right sided weakness with NIH stroke scale of 7 . At that facility, the physicians called for a tele stroke consult and he underwent CT imaging which was negative for any acute abnormalities. Because the patient was within therapeutic window, tissue plasminogen activator (tPA) was offered and patient consented. Right before the medication administration he had an episode of seizure which led to transfer to our facility for possible intervention and advanced management. Repeat imaging in our facility showed loss of gray-white differentiation, most noticeable at the left basal ganglia and anterior temporal lobe suggestive of a left middle cerebral artery (MCA) territory infarct (Figure 1). It also showed vasogenic edema which is unusual after an acute ischemic stroke. CT angiogram of the head and neck did not show any large vessel occlusion. His clinical presentation and atypical CT findings prompted us for further investigation with perfusion images which showed increase cerebral blood flow and volume (Figure 2). These perfusion images were consistent with high flow state or metabolically active area rather ischemic stroke. Taking into account those suspicions, patient underwent MRI of the brain with and without contrast and MR spectroscopy which showed an illdefined infiltrative T2/FLAIR hyperintense lesion with focal nodular enhancement involving left frontal operculum, left insula and left basal ganglia which were concerning for high grade glioma (Figure 3). Neurosurgery was consulted and biopsy showed high grade anaplastic astrocytoma.

\section{Discussion}

High-grade gliomas are malignant, often rapidly progressive brain tumors that are divided into anaplastic gliomas (anaplastic astrocytoma, anaplastic oligodendroglioma, and anaplastic oligoastrocytoma) and glioblastoma multiforme (GBM) based upon their histopathologic features. Patients with high-grade glioma typically present with subacute and progressive neurologic signs and symptoms that vary according to the location of the tumor within the brain. Magnetic resonance imaging (MRI) of the brain provides confirmatory evidence of a mass lesion, but a tissue diagnosis is ultimately required to distinguish high-grade gliomas from other primary and metastatic brain tumors. Most common symptoms at the presentation include headache, memory loss, motor weakness, visual symptoms, personality changes and language deficit. Patients can also present with mental status changes, cranial nerve palsies, myelopathy or cauda equine syndrome if there is meningeal dissemination [1]. Most common acute presentation of brain tumor includes intracranial bleed, seizures and rarely ischemic stroke. Anaplastic astrocytoma presenting as hemorrhagic stroke has been reported in the literature [2].

Many case reports have reported the incidence of ischemic stroke in the setting of glioma specially glioblastoma [3,4]. Postulated theories of this association include vasculopathy, direct vascular infiltration and damage of the vessel wall from previous radiation treatment. Venous clots from hypercoagulable state is a common finding in glioma patients but there is no increased risk of arterial thrombus [5]. In our case, the patient had normal angiogram and there is no enhancement of the arterial wall which excludes all the above possibilities. Brain tumors misdiagnosed as a stroke is common in the pre-CT scan era [6].

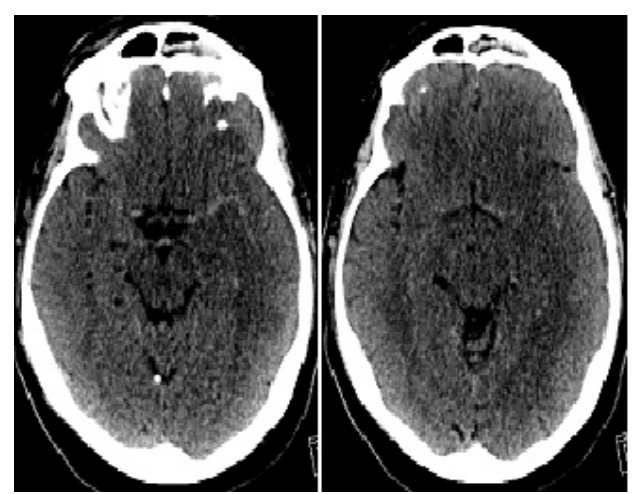

Figure 1: Non contrast head CT showing diffused loss of gray-white matter differentiation in the left hemisphere.
Austin J Cerebrovasc Dis \& Stroke - Volume 4 Issue 2 - 2017 ISSN : 2381-9103 | www.austinpublishing group.com Krishnaiah et al. () All rights are reserved
Citation: Krishnaiah B and Ermak D. Anaplastic Astrocytoma Presenting as Ischemic Stroke: A Diagnostic Pitfall. Austin J Cerebrovasc Dis \& Stroke. 2017; 4(2): 1058. 

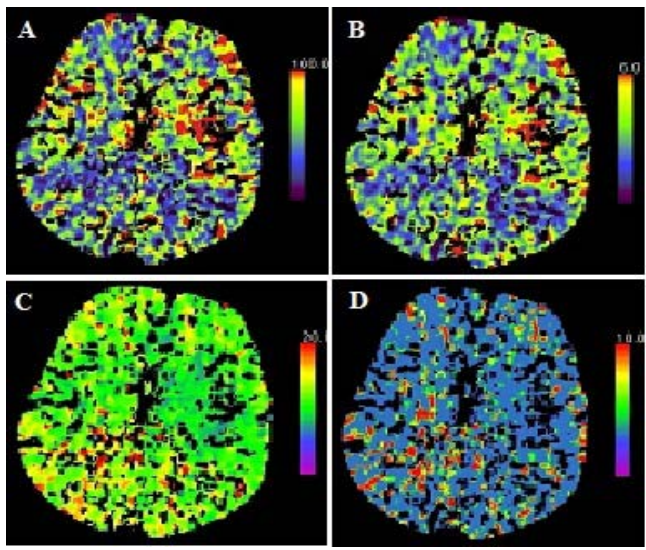

Figure 2: CT perfusion images (A: Cerebral blood flow, B: Cerebral blood volume, C: Time to peak and D: Mean transit time) Perfusion images show increased cerebral blood flow and volume in the left frontal lobe and in the region of the insular cortex.

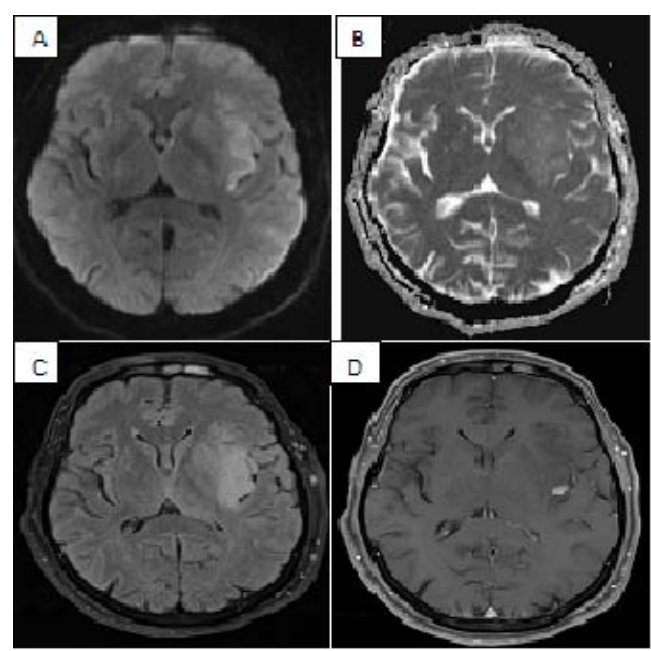

Figure 3: MRI of brain with contrast (A: DWI, B: ADC, C: FLAIR, D: T1 post contrast) showing ill-defined infiltrative FLAIR hyperintense lesion with focal nodular enhancement involving left frontal operculum, left insula and left basal ganglia which were concerning for high grade glioma.

\section{Conclusion}

The misdiagnosis of stroke is more likely to occur in elderly patients given the relative prevalence of stroke in this population, especially when there is no history of neoplasia to suggest otherwise. Similarly, seizure is a well-known complication of acute ischemia and therefore the presence of seizure alone does not rule out an underlying ischemic event. Even with the advent of high end imaging modalities, clinical judgment still remains crucial in diagnosis. In our case, the acute onset symptoms and pre-existing vascular risk factors including diabetes, hypertension and dyslipidemia made ischemic stroke the most likely etiology for the presentation. However, the utility of CT perfusion is well demonstrated in this case whereby focal increase in blood flow and blood volume contradict the diagnosis of stroke and provided the basis for consideration of an alternative diagnosis.

\section{References}

1. Wheen LC, Anderson NE, Baker PC, Singh VK, Synek BJ. Leptomeningeal infiltration as the presenting manifestation of a malignant glioma. J Clin Neurosci. 2006; 13: 298-301.

2. Li L, Yin J, Li Y, Tian W, Qiao B, Tang Z, et al. Anaplastic astrocytoma masquerading as hemorrhagic stroke. J Clin Neurosci. 2013; 20: 1612-1614.

3. Chen H, Cebula H, Schott R, Dietemann JL, Froelich S. Glioblastoma multiforme presenting with ischemic stroke: case report and review of the literature. J Neuroradiol. 2011; 38: 304-307.

4. Pina S, Carneiro Â, Rodrigues T, Samões R, Taipa R, Melo-Pires M, et al. Acute ischemic stroke secondary to glioblastoma. A case report. Neuroradiol J. $2014 ; 27: 85-90$.

5. Marras LC, Geerts WH, Perry JR. The risk of venous thromboembolism is increased throughout the course of malignant glioma: an evidence-based review. Cancer. 2000; 89: 640-646.

6. Morgenstern LB, Frankowski RF. Brain tumor masquerading as stroke. $\mathrm{J}$ Neurooncol. 1999; 44: 47-52.
Austin J Cerebrovasc Dis \& Stroke - Volume 4 Issue 2 - 2017 ISSN : 2381-9103 | www.austinpublishing group.com Krishnaiah et al. (C) All rights are reserved
Citation: Krishnaiah B and Ermak D. Anaplastic Astrocytoma Presenting as Ischemic Stroke: A Diagnostic Pitfall. Austin J Cerebrovasc Dis \& Stroke. 2017; 4(2): 1058 